\title{
Role of prostaglandin E2 receptor 4 in the modulation of apoptosis and mitophagy during ischemia/reperfusion injury in the kidney
}

\author{
CHENGUANG DING ${ }^{1,2^{*}}$, FENG HAN $^{1,2^{*}}$, HELI XIANG ${ }^{1,2^{*}}$, YUXIANG WANG $^{1,2}$, MENG DOU $^{1,2}$,

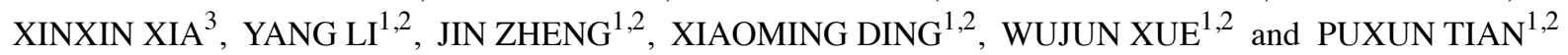 \\ ${ }^{1}$ Department of Kidney Transplantation, Nephropathy Hospital, The First Affiliated Hospital of Xi'an Jiaotong University; \\ ${ }^{2}$ Institute of Organ Transplantation, Xi'an Jiaotong University; ${ }^{3}$ Department of Traditional Chinese Medicine, \\ The First Affiliated Hospital of Xi'an Jiaotong University, Xi'an, Shaanxi 710061, P.R. China
}

Received March 28, 2018; Accepted April 4, 2019

DOI: $10.3892 / \mathrm{mmr} .2019 .10576$

\begin{abstract}
The mechanisms by which prostaglandin E2 receptor 4 (EP4) protects against renal ischemia-reperfusion (I/R) injury (IRI) remain to be fully elucidated. In the present study, the protective effects of EP4 signaling on renal mitochondria and against renal IRI, as well as the underlying mechanisms, were investigated. A rat model of renal IRI was established. The right kidney was separated without damaging the artery clip, and the renal blood perfusion was then restored after $60 \mathrm{~min}$. One group of animals was treated with EP4 agonists prior to I/R. The mitochondrial mass, the copy number of mitochondrial (mt)DNA, adenosine triphosphate (ATP) production and mitochondrial autophagy were analyzed. It was identified that renal IRI reduced the mitochondrial mass, decreased the mtDNA copy number and inhibited ATP production. The loss of renal mitochondria was attributed to the excessive mitochondrial autophagy induced by renal IRI. Pre-treatment with EP4 agonist inhibited excessive mitochondrial autophagy, the loss of mitochondria and maintained and the energy imbalance within the cells. It was indicated that renal IRI causes excessive mitochondrial autophagy, which is one of the important causes of renal dysfunction.
\end{abstract}

\section{Introduction}

The kidneys are the major osmoregulatory organs in the body, which are not only involved in metabolic waste processing and hormone secretion, but also maintain the water-electrolyte and

Correspondence to: Dr Puxun Tian, Department of Kidney Transplantation, Nephropathy Hospital, The First Affiliated Hospital of Xi'an Jiaotong University, 277 West Yanta Street, Xi'an, Shaanxi 710061, P.R. China

E-mail: yuantian@mail.xjtu.edu.cn; xwujun@126.com

${ }^{*}$ Contributed equally

Key words: renal ischemia-reperfusion injury, EP4, mitochondria, autophagy, cAMP, PKA the acid-base balance. Research indicates that neighbor and distant organs to the kidneys, including the heart, lung, liver, intestine and brain, are severely damaged when renal function is impaired (1).

Acute kidney injury (AKI) is a type of rapid renal dysfunction caused by multiple etiological factors and pathological syndromes, and is characterized by a high mortality (1). Kidneys, as hypertransfusion organs, are extremely sensitive to ischemia, and ischemia-reperfusion (I/R) injury (IRI) is common in kidneys (2). IRI is one of the major causes of AKI. Renal IRI is a common pathological and physiological phenomenon. Renal IRI frequently affects patients with hypertension or those who have undergone renal surgery (3). Renal IRI is also a significant factor that leads to delayed functional recovery after kidney transplantation, poor outcomes and even acute renal failure (4). Therefore, strategies to effectively reduce renal IRI in clinical practice are urgently required.

It is known that acute renal failure is significantly associated with renal IRI, but the pathological mechanisms of renal IRI are complex (5). Most of the recent studies on IRI primarily focus on aspects including apoptosis and necrosis of renal tubular epithelial cells, free radical-induced injury, calcium overload, the obstacles of energy metabolism, adhesion molecules and cell factors (6,7). Once IRI has occurred, the prognosis of the patients is significantly affected. However, there is currently a lack of effective drugs. In clinical practice, affected patients are mainly dependent on renal replacement therapies, including hemodialysis and peritoneal dialysis, while passively waiting for the damaged renal function to recover by itself (8). Therefore, the discovery of specific therapeutic targets for treating renal IRI has great potential for clinical application.

The mechanisms of renal IRI are complex, and mainly involve the generation of numerous types of reactive oxygen species, inflammatory damage and disturbance of the microcirculation. The key link is disordered cell energy metabolism. Mitochondria, which produce energy for the cell through oxidative phosphorylation, are an important type of organelle. The regulatory mechanisms of mitochondrial function are closely associated with IRI (9).

Autophagy plays an important role in renal IRI, and may even trigger apoptosis and necrosis. Autophagic cell death is 
a type of non-apoptotic and programmed cell death induced by oxidative stress and injury. Moderate autophagy is necessary for cells to complete their own metabolism and organelle renewal. It is important for maintaining the stability of the body's environment, and for regulating cell damage and aging. Non-physiological and excessive autophagy leads to disproportionate cell death, causing pathological changes in tissues and organs. After reperfusion of renal cells, autophagy is significantly increased. After drug intervention or RNA interference is used to inhibit the expression of autophagy-associated genes, serum creatinine $(\mathrm{sCr})$ and urea nitrogen levels are significantly decreased, and the pathological changes in the kidneys are markedly reduced (10). Kimura et al (11) established a model of IRI in autophagy related 5 (Atg5)-knockout mice and was the first to demonstrate the protective effect of autophagy on renal IRI using genetic evidence in vivo. Research has indicated that an abnormal increase in autophagy in renal cells after reperfusion may be one of the important triggering factors for structural and functional damage of the kidney (12). Therefore, inhibition of autophagy is expected to prevent renal IRI and even apoptosis.

Prostaglandin E2 (PGE2) is an important endogenous regulatory factor in the body. When renal I/R occurs, PGE2 exerts an important role in regulating the inflammatory reaction and microcirculation. Cyclooxygenase-2 (COX-2) is an important rate-limiting enzyme regulating the production of PGE2; it has been proven that COX-2 has an important role in renal IRI (13).

PGE2 has four G protein-coupled receptors, namely prostaglandin E2 receptor (EP)1-4. EP4 is one of the important $\mathrm{G}$ protein-coupled receptors, and is a downstream molecule of COX-2. After activation, EP4 may bind with intracellular $G$ proteins and upregulate the intracellular cyclic (c)AMP concentration to influence the downstream signaling of extracellular signal-regulated kinase $1 / 2$. When IRI occurs, the gene expression of EP4 increases, while that of EP3 decreases, while the expression of EP1 and EP2 is not significantly affected, suggesting that EP4 and EP3 signals may be directly involved in the pathological processes of IRI. Furthermore, EP4 agonists may significantly reduce IRI. Since COX-2 is located upstream of EP4, COX-2 inhibitors suppress the opening of the mitochondrial permeability transition pore during reperfusion by inhibiting the activity of glycogen synthase kinase-3 $\beta$, an important intracellular signaling molecule. Studies on in vitro tumor cells have found that EP4 protein can inhibit cell necrosis and apoptosis. Xiao et al (14) suggested that EP4 signaling is involved in the process of myocardial I/R injury, and the use of EP4 agonists can alleviate myocardial damage. Liang et al (15) found that activation of EP4 signaling can attenuate brain I/R injury. CAY10598 is a commonly used EP4 agonist. In the present study, we used it as a tool. The protective effect of EP4 signaling on IRI is achieved by regulating the mitochondrial function of cells, but this remains to be confirmed.

Although renal transplantation is a frontier discipline, which has developed rapidly in recent years, IRI is an inevitable adverse event associated with it. Current research therefore has focused on the mechanisms of IRI with the aim to identify approaches to significantly inhibit or prevent it. In this light, the present study investigated the protective effect of
EP4 signaling on renal mitochondria and IRI, and investigated the underlying mechanisms in order to provide strategies to reduce IRI in renal transplantation.

\section{Materials and methods}

Experimental grouping. A total of 18 male Sprague Dawley rats (weight, 250-300 $\mathrm{g}$ and age, 9 weeks) were used in the present study. The rats were provided by the Animal Center of Xi'an Jiaotong University. Rats were fed and housed in an animal room at approximately $25^{\circ} \mathrm{C}, 3$ per cage, with a natural circadian rhythm, and a week of free eating. The rats were provided ad libitum access to standard rat chow and water prior to and after the experiment. The animals were randomly divided into three groups, namely the sham group, the I/R group and the I/R+CAY10598 group, with 6 rats in each group. In the Sham group, the abdominal cavity was opened, but the pedicle of the blood vessels was not blocked. In the I/R group, after $60 \mathrm{~min}$ of bilateral renal ischemia, the vascular clamps were opened, resulting in reperfusion, which was sustained for $24 \mathrm{~h}$. In the I/R+CAY10598 group, the rats were intraperitoneally injected with $1 \mathrm{mg} / \mathrm{kg}$ EP4 agonist CAY10598 at $1.5 \mathrm{~h}$ prior to IRI, which was performed as in the I/R group. All protocols were approved by the Institutional Animal Care and Use Committee of the Xi'an Jiaotong University (Xi'an, China) (Permit no. XJTULAC2016-558).

Rat model of renal IRI. The animals were fasted overnight prior to the experiments with free access to water. They were anesthetized by an intraperitoneal injection of $300 \mathrm{mg} / \mathrm{kg}$ $10 \%$ chloral hydrate. A medial incision was performed in the back, and the renal as well as the bilateral renal arteries were separated. Non-invasive dissection of the artery clip was quickly performed for the bilateral renal arteries at $60 \mathrm{~min}$ after opening the artery clip, and blood flow perfusion was restored, followed by suturing of the incision.

Primary culture and cell viability determination of rat renal tubular epithelial cells. After establishing the rat model of renal IRI, anaesthetic was intraperitoneally injected $(10 \%$ chloral hydrate at $300 \mathrm{mg} / \mathrm{kg}$ ). The rats were then sacrificed by cervical dislocation and placed in $75 \%$ alcohol for $30 \mathrm{sec}$. After the rats were fixed on the operating table, the abdominal cavity was opened with surgical scissors and a surgical fistula, and the bilateral renal were taken out and placed in pre-cooled phosphate-buffered saline (PBS). The renal cortex was obtained and cut into small pieces, and the resulting mixture was filtered through an 80-mesh filter. The supernatant was filtered through a 100-mesh filter to leave the tissue block. The cells were mixed with PBS, centrifuged for $5 \mathrm{~min}$ at 1,200 rpm (300 x g), the supernatant was discarded, and collagenase IV (C5138; Sigma-Aldrich; Merck KGaA, Darmstadt, Germany) $(1 \mathrm{mg} / \mathrm{ml})$ was added to the cells, followed by incubation at $37^{\circ} \mathrm{C}$ for $15 \mathrm{~min}$. Following centrifugation (with the aforementioned conditions), the supernatant was discarded, and the cells were suspended in serum-containing Dulbecco's modified Eagle's F12 medium (12634-010; Thermo Fisher Scientific, Inc., Waltham, MA, USA). The cells from each group were then inoculated in a 96-well plate at $5.0 \times 10^{4}$ cells in $100 \mu \mathrm{l} /$ well. After $24 \mathrm{~h}$ of culture, the medium was discarded 
and $10 \mu \mathrm{l}$ of Cell Counting Kit-8 (CCK-8) (cat. no. 96992 , USA, Sigma-Aldrich; Merck KGaA) solution was added to each well. After further incubation at $37^{\circ} \mathrm{C}$ for $4 \mathrm{~h}$, the light absorbance was measured at a wavelength of $450 \mathrm{~nm}$ using a microplate reader, and the relative viability of the cells was calculated.

Determination of $s C r, \beta 2$-microglobulin $(\beta 2-M G)$ and neutrophil gelatinase-associated lipocalin (NGAL). $\mathrm{sCr}$ (E-EL-S1181c), as well as serum concentrations of $\beta 2-\mathrm{MG}$ (E-EL-R1085c) and NGAL (E-EL-R0662c), were determined using ELISA kits (Elabscience, Wuhan, China) according to the manufacturer's instructions.

Hematoxylin and eosin $(H \& E)$ staining. For histopathological analysis, the renal tissues were paraffin-embedded and sectioned (5-mm in thickness), followed by stepwise staining. In brief, the sections underwent dewaxing, gradient alcohol hydration, washing, hematoxylin staining, washing, eosin staining, gradient alcohol dehydration, transparency and mounting/sealing with neutral resin. Finally, the pathological changes in the kidneys were observed under an optical microscope.

ATP determination. Renal tissue (100 mg) was accurately weighed and $1 \mathrm{ml}$ pre-cooled ATP lysis solution was added, followed by homogenization using an electric homogenizer. The tissue homogenate was transferred to a $1.5-\mathrm{ml}$ centrifuge tube, and centrifuged at $4^{\circ} \mathrm{C}$ and $12,000 \times \mathrm{g}$ for $10 \mathrm{~min}$. The supernatant remained. A total of $100 \mu \mathrm{l}$ of the sample was added to each well of a microtiter plate, and $100 \mu$ ATP assay solution was added. Following mixing, the BioTek Synergy 4 Multiplate reader (BioTek Instruments, Inc., Winooski, VT, USA) was immediately employed to determine the relative light units. According to the standard curve, the ATP content of each sample was interpolated.

JC-1 assay. The mitochondrial membrane potential of the renal tubular epithelial cells was assessed using the Flow Cytometry Mitochondrial Membrane Potential Detection kit (cat. no. 551302; BD Biosciences, Franklin Lakes, NJ, USA). For each sample, $1 \times 10^{6}$ cells were transferred to a flow cytometry tube and $400 \mu 1$ binding buffer was added. The diluted conditioned mitochondria were added to the tested mitochondria at a volume ratio of 9:1. Following agitation, the cells were incubated at $37^{\circ} \mathrm{C}$ for $15 \mathrm{~min}$, re-suspended and analyzed using a flow cytometer (BD Biosciences). The fluorescence intensity in A.U. was determined for each sample.

Detection of mitochondrial (mt)DNA fragments. Total DNA was isolated from the cytologic fractions prepared from the renal tissues using a DNA extraction kit (Qiagen, Hilden, Germany). The amount of mtDNA was determined by detecting NADH dehydrogenase subunit 1 (NDl) with $\beta$-actin as the housekeeping gene. The $\mathrm{C} 1$ mean difference between the reference gene and the test sample was calculated. Finally, the ratio of the ND1 gene and the nuclear $\beta$-actin gene copy was obtained.

Determination of glutathione (GSH), malondialdehyde (MDA), superoxide dismutase (SOD) and COX levels by ELISA. The content of GSH (cat. no. HS3275; Luzheng,
Shanghai, China), MDA (cat. no. HPBIO; Hepeng, Shanghai, China), SOD (cat. no. BMS222; Biosource; Thermo Fisher Scientific, Inc.) and COX (cat. no. HS2185; Luzheng) in renal tissues was determined by double antibody sandwich ELISA. The procedures were performed in strict accordance with the manufacturer's protocols. The measurement of each sample and standard product was performed in 3 replicates. The absorbance was determined at a wavelength of $450 \mathrm{~nm}$. The sample concentration was determined based on the standard curve.

Microtubule-associated protein 1-light chain $3 B$ (LC3B) immunofluorescence analysis. The cells were washed with PBS, fixed with $4 \%$ paraformaldehyde for $10 \mathrm{~min}$ at room temperature, washed and then incubated with $0.1 \%$ Triton $\mathrm{X}-100$ for $5 \mathrm{~min}$. After blocking for $1 \mathrm{~h}$ at room temperature, the cells were incubated with anti-LC3B (dilution 1:100; cat. no. 700712; Invitrogen; Thermo Fisher Scientific, Inc.) in the dark for $2 \mathrm{~h}$ at room temperature, followed by incubation with the fluorescein isothiocyanate-conjugated antibody (cat. no. 715-096-151, donkey anti-mouse; dilution 1:100; Jackson ImmunoResearch Laboratories, Inc., West Grove, PA, USA) in the dark for $30 \mathrm{~min}$ at room temperature. The cells were washed and counterstained with DAPI for $1 \mathrm{~min}$. Subsequently, the cells were mounted with buffered glycerol and observed under a fluorescence microscope (magnification x200).

Western blot analysis. Lysates were prepared from renal tissues using ice-cold lysis buffer containing a cocktail of protease inhibitors (Sigma-Aldrich; Merck KGaA). Crude protein lysates $(50 \mu \mathrm{g})$ were separated by $12 \%$ SDS-PAGE, followed by transfer to a nitrocellulose membrane (Bio-Rad Laboratories, Inc., Hercules, CA, USA) and blocking for $2 \mathrm{~h}$ with 5\% non-fat powdered milk dissolved in Tris-buffered saline with $0.05 \%$ Tween-20 at room temperature with agitation. The membranes were then incubated overnight at $4^{\circ} \mathrm{C}$ with antibodies to the following proteins: Cytochrome $c$ (1:100, cat. no. ab133504), caspase-3 (1:100, cat. no. ab197202), caspase-9 (1:100, cat. no. ab219590), LC3BII (1:200, cat. no. ab192890), Atg5 (1:100, cat. no. ab108327), lysosomal-associated membrane protein (LAMP)1 (1:200, cat. no. ab108597), P62 (1:100, cat. no. ab109012), protein kinase A (PKA) (1:100, cat. no. ab32390), phosphorylated cAMP response element binding protein (p-CREB) $(1: 200$, cat. no. ab32096), aquaporin 5 (AQP5) (1:100, cat. no. 1b92320) (all from Abcam, Cambridge, MA, USA) and anti- $\beta$-actin (dilution 1:5,000; Sigma-Aldrich; Merck KGaA). Anti-mouse horseradish peroxidase-conjugated secondary antibodies (cat. no. A8592, Sigma-Aldrich; Merck $\mathrm{KGaA}$ ) were used at 1:3,000 dilution. Immunoreactive bands were visualized using a diaminobenzidine kit (Zhongshan Golden Bridge Biotechnology Co., Ltd., Beijing, China).

Statistical analysis. All experiments were repeated 3 times. Values are expressed as the mean \pm standard error of the mean. One-way analysis of variance (ANOVA) was used for comparison between groups, and least significant difference (LSD) or Student-Newman-Keuls (SNK) tests were used for comparison between groups. Analysis of variance was performed to assess the differences among multiple groups using GraphPad Prism 6.0 software (GraphPad Software, Inc., La Jolla, CA, USA). 
A

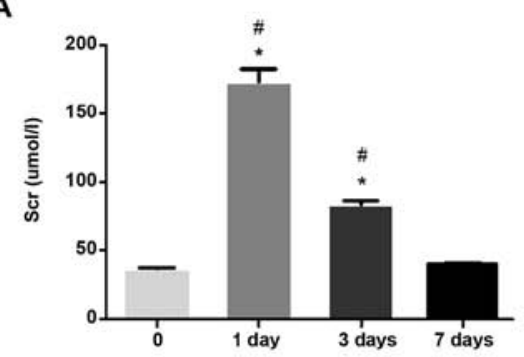

D

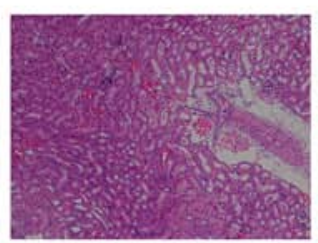

Sham

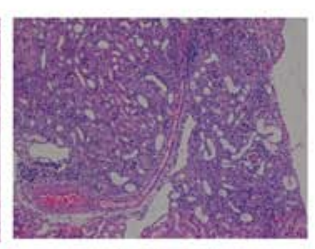

I/R model
B
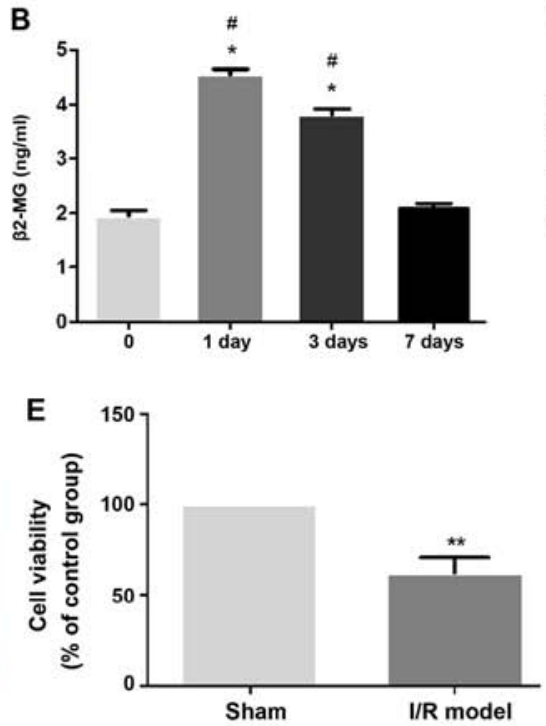

C

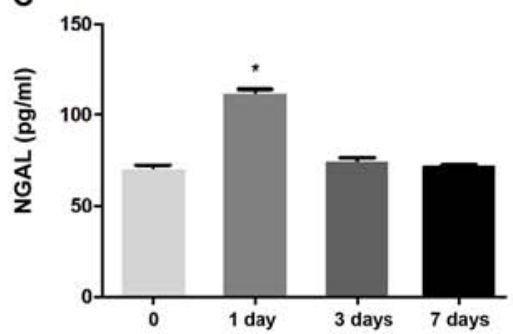

$\mathbf{F}$

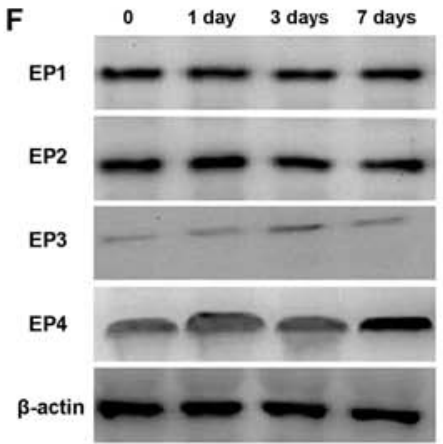

Figure 1. (A-C) Kidney function parameters (sCR, $\beta 2-\mathrm{MG}$ and NGAL) in the serum of all groups in renal I/R injury. Values are expressed as the means \pm SD for group. ${ }^{*} \mathrm{P}<0.05$ indicates a significant difference when compared to the 0 day group; ${ }^{\#} \mathrm{P}<0.05$ indicates a significant difference when compared to the day 7 group. (D) H\&E staining showed an increased tubular necrosis in renal I/R injury compared with the Sham group. Original magnification, $\mathrm{x} 100$. (E) Compared with the Sham group, renal tubular epithelial cell viability was significantly decreased after $I / R ;{ }^{* *} \mathrm{P}<0.01$. (F) In the early stage of renal $\mathrm{I} / \mathrm{R}$, the expression of EP4 exhibited only a minor change, but with the prolongation of time, the expression of EP4 protein was significantly increased on day 7 after surgery. EP4, prostaglandin E2 receptor 4; I/R, ischemia-reperfusion; sCr, serum creatinine; NGAL, neutrophil gelatinase-associated lipocalin; $\beta 2$-MG, $\beta 2$-microglobulin.

$\mathrm{P}<0.05$ was considered to indicate a significant difference between groups.

\section{Results}

Impaired renal function and altered EP4 in renal IRI. Markers of renal function in the different groups are provided in Fig. 1. The levels of $\mathrm{sCr}$, as well as serum $\beta 2-\mathrm{MG}$ and NGAL, were significantly increased in the IRI rats compared with those on day 0 (pre-operative; $\mathrm{P}<0.05$ ). Within the same group, markers of renal function gradually decreased with time (Fig. 1A-C). Histopathology confirmed that in the I/R group, numerous renal tubular epithelial cells were necrotic, and interstitial congestion and edema were observed (Fig. 1D). The cell viability assay confirmed that compared with the Sham group, the viability of renal tubular epithelial cells was significantly decreased after I/R (Fig. 1E). When the expression of EP1-4 proteins was examined, it was identified that EP3 and EP4 exhibited minor changes after I/R. The expression of EP4 protein was significantly increased on day 7 after surgery (Fig. 1F).

EP4 agonist CAY10598 inhibits mitochondrial membrane potential, cytochrome c release and apoptosis associated with renal IRI. Ischemia-reperfusion injury can cause excessive production of reactive oxygen species, calcium overload, mitochondrial damage and cell death. Excessive reactive oxygen species, as a stimulation of apoptosis, triggers the mitochondrial apoptotic pathway, leading to the release of apoptotic factors such as cytochrome $c$. Once released into the cytosol, cytochrome $c$ activates the downstream caspase family (16). Compared with that in the Sham group, the mitochondrial membrane potential in the I/R group was significantly decreased $(\mathrm{P}<0.001)$. However, administration of CAY10598 prior to the induction of IRI inhibited this significant decrease in the mitochondrial membrane potential $(\mathrm{P}<0.01$; Fig. 2A and B). The levels of cytochrome $c$, caspase-3 and caspase-9 were significantly increased in the I/R group compared with those in the Sham group $(\mathrm{P}<0.01)$. The levels of cytochrome $c$, caspase- 3 and caspase- 9 were significantly decreased in the I/R+CAY10598 group compared with these levels in the I/R group (Fig. 2C-H). Thus, treatment with CAY10598 reversed the I/R-mediated increase in cytochrome $c$, caspase-3 and caspase- 9 .

EP4 agonist CAY10598 has a protective effect on mitochondrial dysfunction after renal IRI. As depicted in Fig. 3, I/R promoted a significant decrease in renal levels of ATP, mtDNA, GSH, SOD and COX and a significant increase in MDA. A significant increase was noted in the renal levels of ATP, mtDNA, GSH, SOD and COX in the I/R+CAY10598 group compared with the levels in the I/R group. The opposite trend was observed for the content of MDA. Thus, CAY10598 rescued the I/R-promoted decrease in ATP, mtDNA, GSH, SOD and COX and increase in MDA suggesting the protective effect of the EP4 agonist on IRI.

EP4 agonist CAY10598 inhibits mitochondrial autophagy after renal IRI. As depicted in Fig. 4A, after autophagy, microtubule-associated protein 1 light chain 3B II (LC3BII) is transferred from the nucleus to the cell membrane. At the protein level, the expression of LC3BII was significantly increased in the I/R group. Pre-treatment with CAY10598 reduced the I/R-mediated increase in LC3BII in renal tissues of the I/R+CAY10598 group compared with the model I/R group 
A

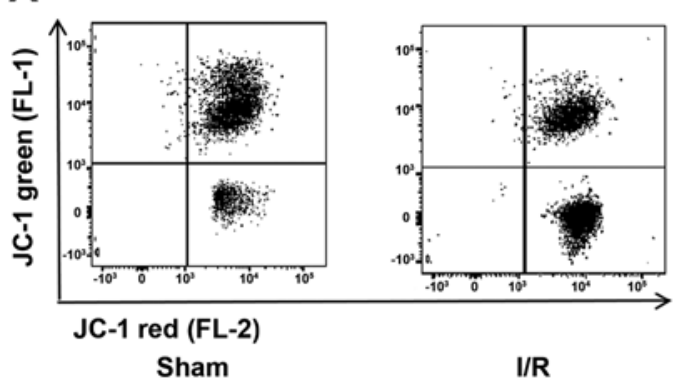

C

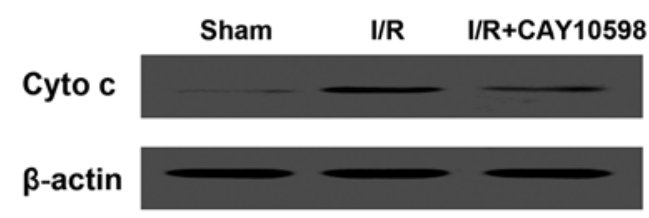

E

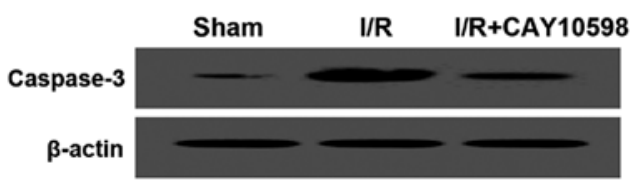

G

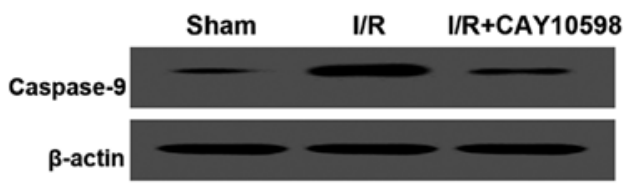

B

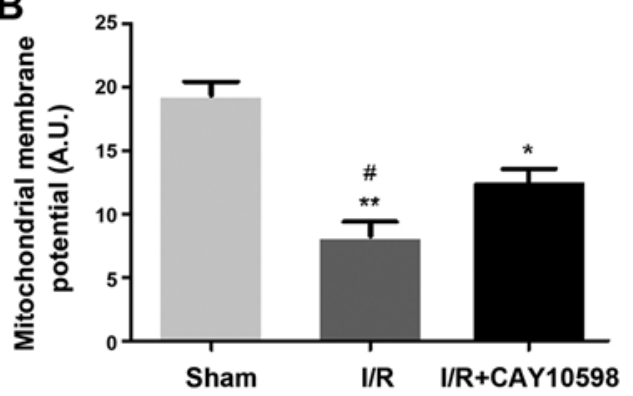

D

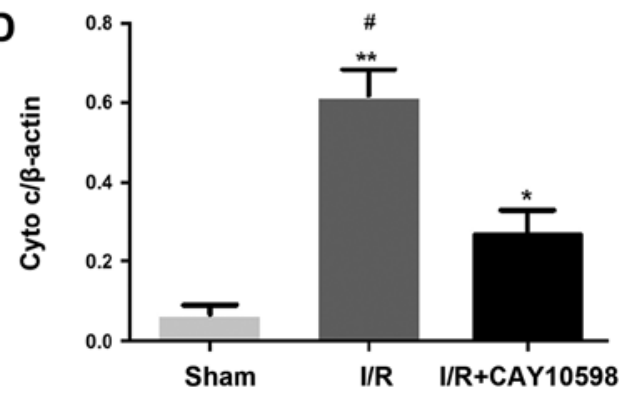

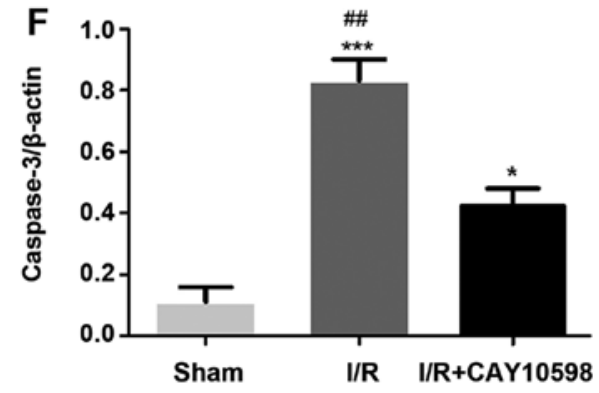

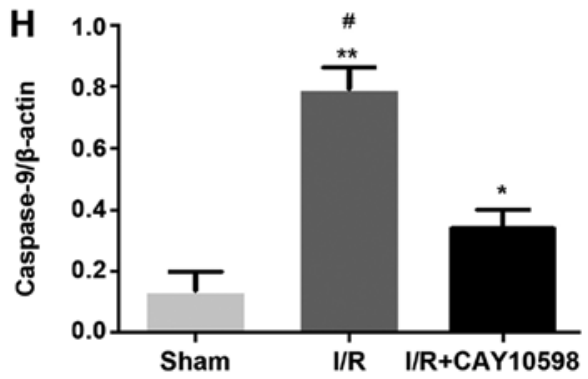

Figure 2. EP4 agonist CAY10598 reverses mitochondrial membrane potential downregulation, cyto $c$ release and increased apoptosis increase following renal I/R injury. (A and B) FACS confirmed that compared with the Sham group, mitochondrial membrane potential in the I/R injury group was significantly decreased which was significantly rescued by pre-treatment with CAY10598. ${ }^{*} \mathrm{P}<0.05,{ }^{* *} \mathrm{P}<0.01$ vs. the Sham group; ${ }^{*} \mathrm{P}<0.05$ vs. the $\mathrm{I} / \mathrm{R}+\mathrm{CAY} 10598$ group. Western blot analysis showed that I/R induced an increase in cyto $c$ (C and D); caspase-3 (E and F); caspase-9 (G and H) expression, while CAY10598 is able to suppress the above changes. ${ }^{*} \mathrm{P}<0.05,{ }^{* *} \mathrm{P}<0.01,{ }^{* * * *} \mathrm{P}<0.001$ vs. the Sham group; ${ }^{\#} \mathrm{P}<0.05,{ }^{\# \#} \mathrm{P}<0.01$ vs. the I/R+CAY10598 group. EP4, prostaglandin E2 receptor 4; cyto $c$, cytochrome $c$; I/R, ischemia-reperfusion.

(Fig. 4B and C). The expression levels of autophagy-associated factors, Atg5 and LAMP1 were significantly increased following I/R but this I/R-promoted increased was significantly abrogated in the IRI rats pre-treated with CAY10598 compared with that in the model I/R group (Fig. 4D-G). The opposite trends were observed for the levels of P62 (Fig. 4H and I).

EP4 protects mitochondria from autophagy by activating the $c A M P / P K A$ signaling pathway. I/R promoted the decrease in the levels of cAMP, PKA, p-CREB and AQP5. Administration of CAY10598 prior to I/R resulted in a significant reversal of the I/R-mediated decrease in these levels. Thus an increase in the levels of cyclic adenosine monophosphate (cAMP), protein kinase A (PKA), phosphorylated cAMP response element-binding protein (p-CREB) and aquaporin 5 (AQP5) was observed in the I/R+CAY10598 group compared with the levels in the $\mathrm{I} / \mathrm{R}$ rats $(\mathrm{P}<0.01$; Fig. 5).

Blockage of the $C A M P / P K A$ signaling pathway reduces the inhibitory effect of CAY10598 on mitochondrial autophagy after I/R. Pre-treatment with PAK inhibitor Rp-8-Br-cAMP and CAY10598 significantly decreased cAMP and PKA levels compared with those in the group treated with CAY10598 alone (Fig. 6A and B). Furthermore, the expression levels of 

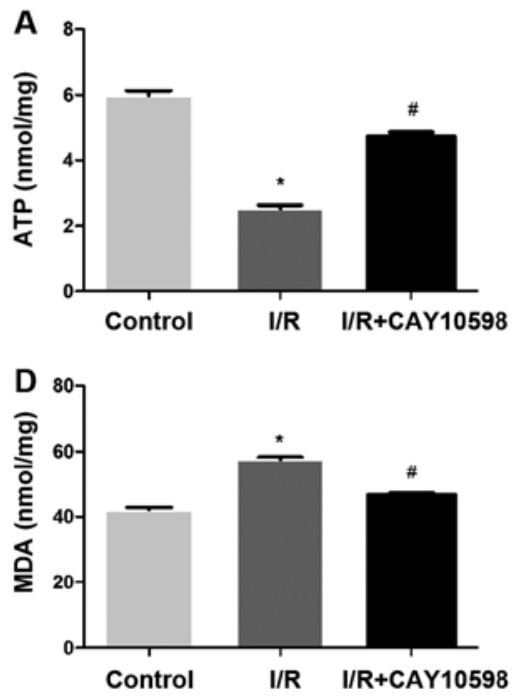
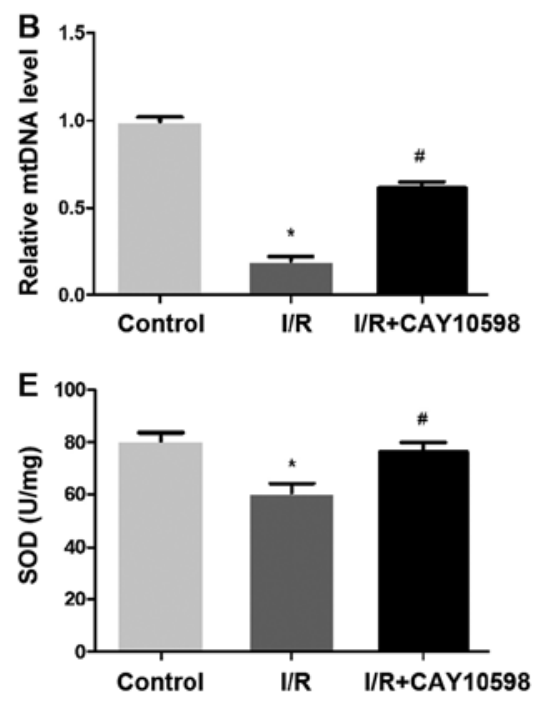
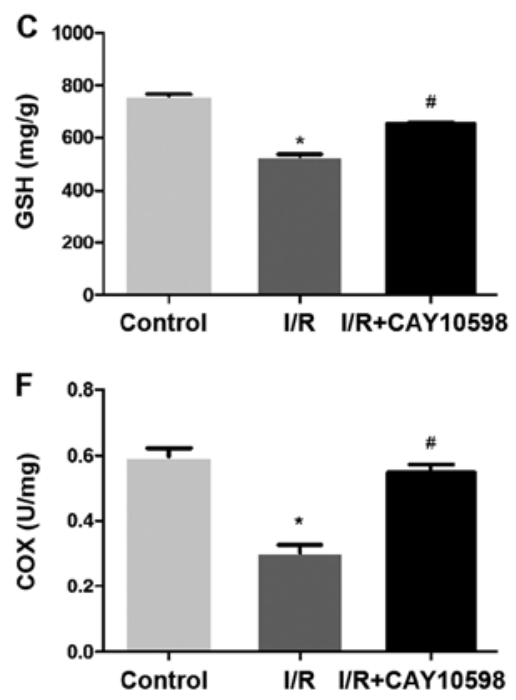

Figure 3. EP4 agonist CAY10598 has a protective effect on mitochondrial dysfunction after renal I/R injury. Levels of (A) ATP, (B) mtDNA, (C) GSH, (D) MDA, (E) SOD and (F) COX in renal tissues of the all experimental groups of rats. ${ }^{*} \mathrm{P}<0.05$ vs. the control group; ${ }^{*} \mathrm{P}<0.05$ vs. the $\mathrm{I} / \mathrm{R}$ group. EP4, prostaglandin E2 receptor 4; I/R, ischemia-reperfusion; adenosine triphosphate (ATP); mitochondrial DNA (mtDNA); glutathione (GSH); malondialdehyde( MDA); superoxide dismutase (SOD); cyclooxygenase (COX).

A
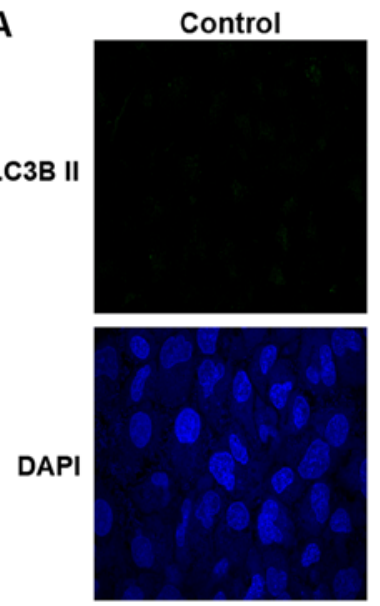

D
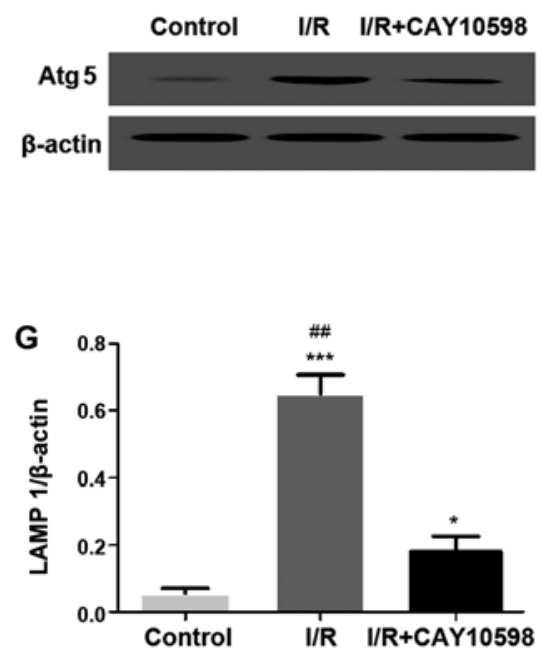

I/R
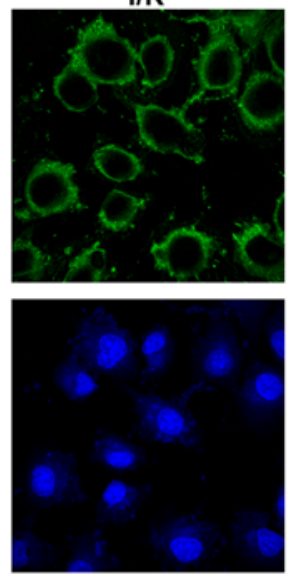

I/R+CAY10598
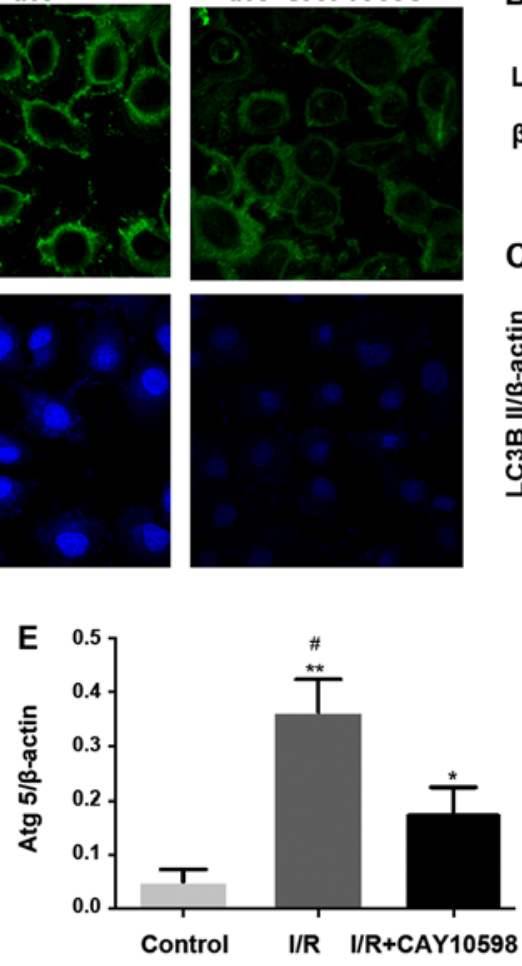

H

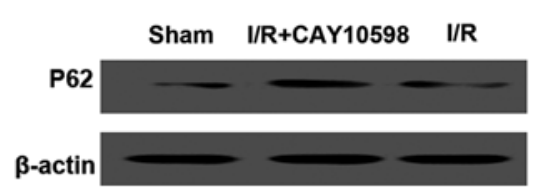

B
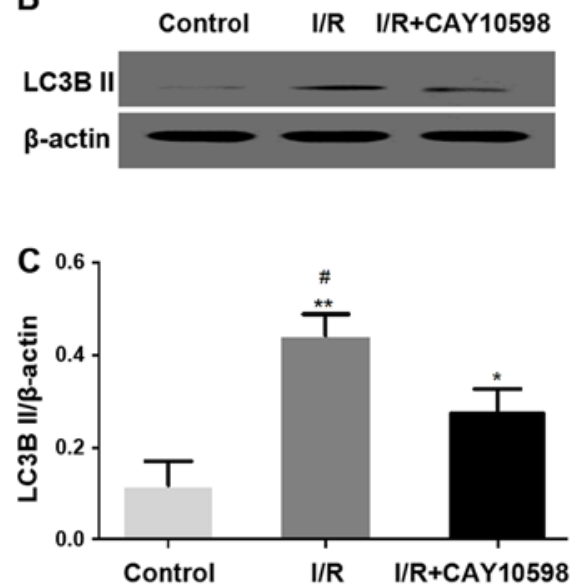

$\mathbf{F}$
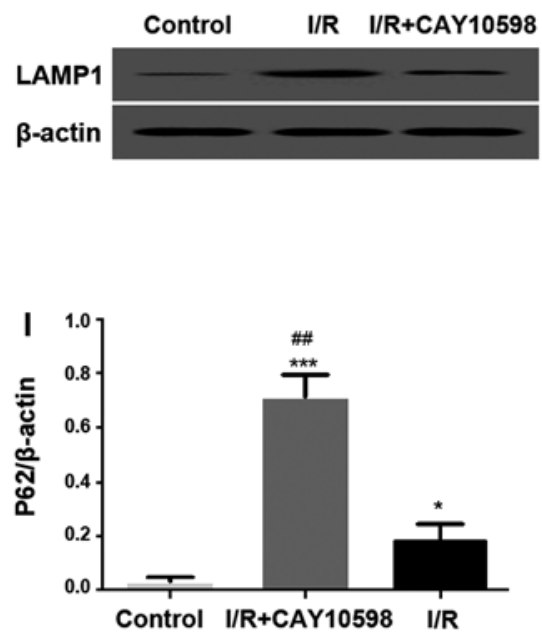

Figure 4. EP4 agonist CAY10598 inhibits mitochondrial autophagy after renal I/R injury. (A) Representative image of immunofluorescence staining confirmed that LC3B II was transferred from the nucleus to the cell membrane in the I/R injury group. Magnification, $\mathrm{x} 400$. Expression levels of LC3BII (B and C), Atg5 (D and E), LAMP1 (F and G) and P62 (H and I) in the renal tissue of all groups of rats. ${ }^{*} \mathrm{P}<0.05,{ }^{* * *} \mathrm{P}<0.01,{ }^{* * *} \mathrm{P}<0.001$ vs. the Sham group; ${ }^{*} \mathrm{P}<0.05$, ${ }^{* \#} \mathrm{P}<0.01$ vs. the I/R+CAY10598 group. EP4, prostaglandin E2 receptor 4; I/R, ischemia-reperfusion; LC3BII, microtubule-associated proteins light chain 3B; Atg5, autophagy related 5; LAMP1, lysosome associated membrane protein-1. 
A

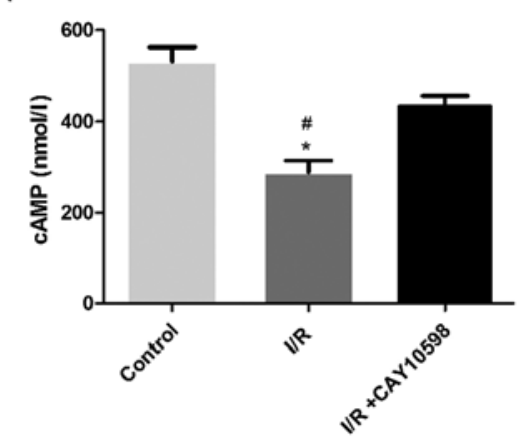

D
B

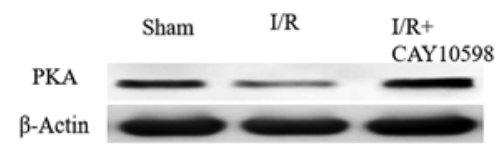

C

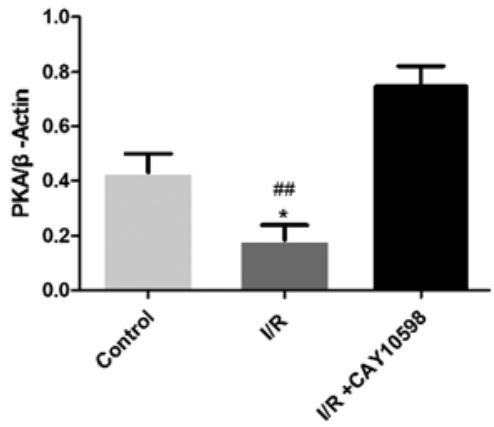

$\mathrm{E}$
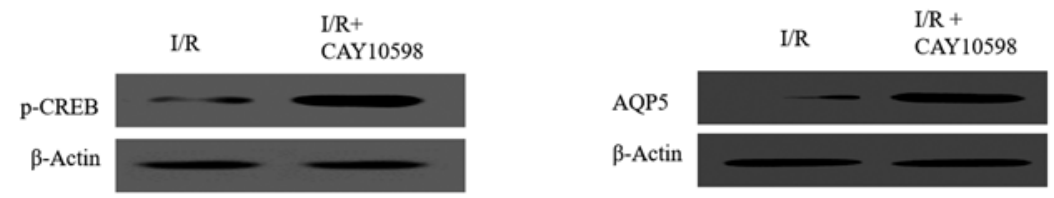

Figure 5. EP4 protects mitochondria from autophagy by activating the cAMP/PKA signaling pathway. Pre-administration of CAY10598 prior to I/R injury resulted in significant increases in the levels of (A) cAMP, (B and C) PKA, (D) p-CREB and (E) AQP5 compared to I/R rats. " $P<0.05$ vs. the Sham group; ${ }^{\text {"}} \mathrm{P}<0.05,{ }^{\# /} \mathrm{P}<0.01$ vs. the I/R+CAY10598 group. EP4, prostaglandin E2 receptor 4; I/R, ischemia-reperfusion; cAMP, cyclic adenosine monophosphate; PKA, protein kinase A; AQP5, aquaporin 5; p-CREB, phosphorylated cAMP response element-binding protein.

autophagy-associated factors LC3BII and P62 were decreased compared with those in the group treated with CAY10598 alone. The expression of Atg5 and LAMP1 were opposite to that of the above proteins (Fig. 6C-F).

\section{Discussion}

Ischemia-reperfusion (I/R) injury (IRI) is a common clinicopathological phenomenon, and in severe cases, it may affect the prognosis of patients. As a common reason for acute kidney injury, renal IRI is also one of the major factors influencing a kidney transplant patient's functional recovery in the early stage and long-term survival (17). Thus, the mechanism of renal IRI requires elucidation in order to develop strategies to improve or reverse IRI. The mechanism of IRI is complex and there are currently no effective measures for its prevention. It has been suggested that IRI is closely linked to cell necrosis, apoptosis and autophagy caused by energy metabolism disorders (18).

Kidney damage mainly manifests as impaired renal function and pathological changes in renal tissue morphology. Renal function is mainly assessed by the $\mathrm{sCr}$ level, and dysfunction of glomerular filtration may cause an elevated sCr. Levels of $\beta 2$-microglobulin ( $\beta 2-\mathrm{MG})$ and neutrophil gelatinase-associated lipocalin (NGAL) are also common parameters used in the evaluation of renal function, with higher levels of $\beta 2-\mathrm{MG}$ and NGAL indicating more severe damage in regards to renal function (19). The present study indicated that serum levels of $\mathrm{Cr}, \beta 2-\mathrm{MG}$ and NGAL in the I/R group were significantly increased compared with the pre-operative levels, indicating that renal dysfunction was present in this group and the IRI model was successfully established. sCr, $\beta 2-\mathrm{MG}$ and NGAL levels gradually declined with time, indicating that renal function was gradually restored. Histopathological changes may reflect the severity of tissue damage (14), and in the present study, morphological changes in the kidney tissue were mainly assessed by histopathological H\&E staining. The results of the present study suggest that compared with those at baseline, levels of $\mathrm{sCr}, \beta 2-\mathrm{MG}$ and NGAL were increased significantly after renal IR, and the increase on day 1 was most obvious. This result validated the successful establishment of the model. On this basis, the morphological changes in renal tissues of rats after renal $\mathrm{I} / \mathrm{R}$ were observed. The results indicated that the rats in the sham-operated group had a clear renal tissue structure, and the renal tubular epithelial cells were intact and well-arranged. In the model group, inflammatory cell infiltration was observed and renal tubular epithelial cells were significantly damaged, while the glomeruli were not obviously affected, indicating renal tissue damage and inflammatory cell infiltration after IRI.

The role of prostaglandin E2 receptor 4 (EP4), which is an important downstream regulatory molecule of prostaglandin E2 signaling in vivo, in renal IRI remains to be fully elucidated $(20,21)$. It has been indicated that EP4 agonists are able to reduce myocardial IRI, but the exact mechanism remains to be determined. It has also been suggested that EP4 agonists modulate the inflammatory response and reduce liver damage. In the present study, it was revealed that the expression of EP4 was significantly decreased after IRI. Furthermore, the expression of EP4 protein was significantly increased on the day 7 after surgery.

Autophagy is a process in which cells degrade their own damaged organelles by lysosomal enzymes under the action of autophagy-associated genes, and it participates in a variety of pathological and physiological activities within the cell $(22,23)$. In 2010, Jiang et al (24) constructed a mouse model of renal IRI. It was confirmed that autophagy has a protective role in renal IRI. In 2011, Kimura et al (11) reported that after IRI in mice lacking the autophagy-associated gene Atg5, the amount of renal tubular epithelial cell apoptosis was significantly 


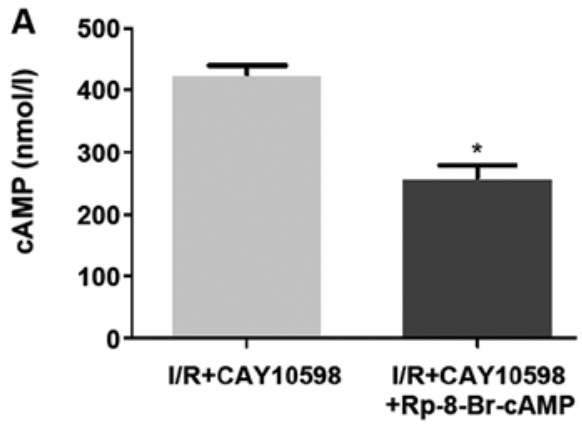

B

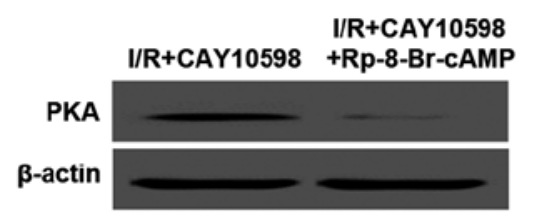

C

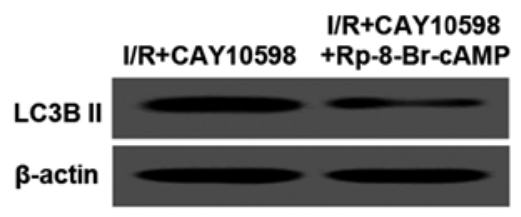

D

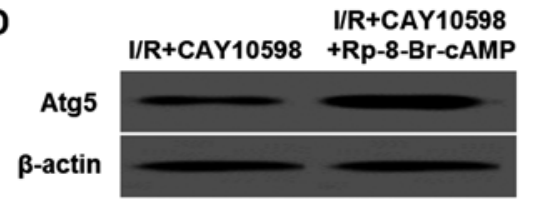

E

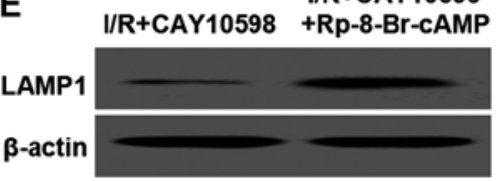

$\mathbf{F}$

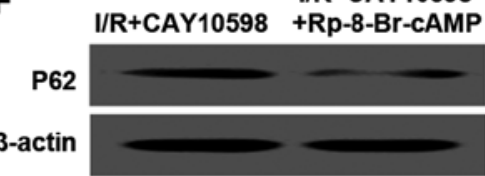

Figure 6. Blockade of the cAMP/PKA signaling pathway reverses the inhibitory effect of CAY10598 on mitochondrial autophagy after I/R injury. Pre-administration of Rp-8-Br-cAMP and CAY10598 prior to I/R injury showed that the expression levels of (A) cAMP, (B) PKA, (C) LC3BII, (D) Atg5,

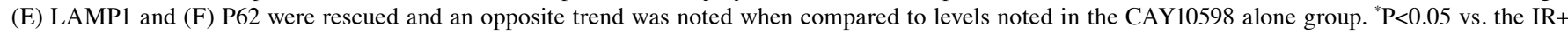
CAY10598 group. cAMP, cyclic adenosine monophosphate; PKA, protein kinase A; LC3BII, microtubule-associated proteins light chain 3B; Atg5, autophagy related 5; LAMP1, lysosome associated membrane protein-1.

higher than that in normal mice, indicating that autophagy maintains the homeostasis of renal tubular epithelial cells and protect against renal IRI. The results of the present study suggested that the EP4 agonist CAY10598 inhibited mitochondrial membrane potential decreases, as well as cytochrome $c$ release and apoptosis following renal IRI.

ATP is the most direct source of energy in the body. The mitochondrial ATP content is an important indicator of mitochondrial function (25). The present study indicated that the expression of EP4 was inhibited during renal IRI, and furthermore, collapse of the mitochondrial membrane potential and $\mathrm{O}_{2}$ utilization disorders may have significantly reduced ATP synthesis. After pre-treatment with EP4 agonist, the mitochondrial ATP content was significantly increased, suggesting that EP4 is beneficial for maintaining mitochondrial ATP (26). A normal number, structure and function of mitochondria are required for the maintenance of the biological repair of the mitochondria. mtDNA regulates the expression of mitochondrial structural and functional proteins, and thus, it plays a crucial role in maintaining the integrity of mitochondrial morphology and function (27-29). The amount of mtDNA reflects the mitochondrial bioremediation function. The results of the present study indicated that the renal mitochondria were damaged by renal IRI and that the copy number of mtDNA was decreased. This result may illustrate that IRI of the kidney may lead to impairment of mitochondrial bio-regeneration. In the group pre-treated with EP4 agonists, the mtDNA copy number gradually recovered. This indicated that EP4 has a protective role to sustain the mitochondrial bio-repair function in renal tubular epithelial cells damaged by renal IRI.

Mitochondrial autophagy is a mechanism of mitochondrial degradation under various stress conditions, which has been demonstrated to occur in a variety of mitochondrial dysfunction-associated diseases (30). Renal IRI leads to mitochondrial damage in renal tubular epithelial cells (31). Activation of caspase-associated signaling leading to cell death has been confirmed in the above studies. In the present study, mitochondrial autophagy in renal IRI was further explored. Detection of autophagy-associated factors revealed excessive mitochondrial autophagy in renal IRI. The results also indicated that mitochondrial autophagic death was attributed to excessive mitochondrial damage. IRI may damage the mitochondria directly or indirectly, thus inducing excessive mitochondrial autophagic clearance. However, the activation of mitochondrial autophagy as a secondary response to mitochondrial damage induced by renal IRI cannot be excluded. In turn, the loss of mitochondrial function may further exacerbate mitochondrial autophagy, which may induce further mitochondrial loss. This self-amplifying circle continues until the cells die. This is probably why inhibition of mitochondrial autophagy is protective in the initial stage, whereas accumulation of damaged mitochondria later in life promotes cell death $(32,33)$.

Finally, the present study further explored the signaling mechanism by which EP4 regulates mitochondrial autophagy. The detection of cAMP levels and PKA signaling downstream of EP4 revealed that IRI reduces the cAMP concentration and the expression of proteins associated with the PKA signaling pathway, while EP4 agonist CAY10598 inhibited this phenomenon (34). The study found that the cAMP-PKA signaling pathway did not only mediate the trafficking and gating of AQPs, but also mediated its redistribution, and found that this signaling pathway regulates the expression pathway of AQP5 which may be related to the CREB transcription initiation site upstream of the AQP5 open reading window; point-related, suggesting that the cAMP/PKA-CREB transcriptional response may be involved in the expression of AQP5 through this site. Stimulated by certain factors, CREB is phosphorylated, cAMP is activated, and the amount of cAMP in the cells is increased, and then PKA is activated. PKA further catalyzes the phosphorylation of serine on CREB, which makes AQP5 highly expressed, and finally increases the permeability of the cell membrane to water. In the present 
study, an increase in AQP5 expression was also detected after CAY10598 pre-treatment. In addition, the results of the present study also indicated that EP4 protected the mitochondria from autophagy by activating the cAMP/PKA signaling pathway, while blockage of the cAMP/PKA signaling pathway reversed the inhibitory effect of CAY10598 on mitochondrial autophagy after IRI. These results confirmed that EP4 protects the kidneys against IRI, and this effect may be achieved by activating the cAMP/PKA signaling pathway and eventually inhibiting excessive mitophagy.

In conclusion, the present study demonstrated the protective effect of EP4 signaling on mitochondria and renal IRI, as well as the underlying mechanisms. It was revealed that renal IRI reduced the renal mitochondrial mass, decreased the copy number of mtDNA and inhibited ATP production. The loss of mitochondria was attributed to the excessive mitochondrial autophagy induced by renal IRI. The EP4 agonist inhibited excessive mitochondrial autophagy, the loss of mitochondria and energy imbalances within the cell. This further confirms that excessive mitochondrial autophagy is instigated by renal $\mathrm{I} / \mathrm{R}$, which is one of the important causes of renal dysfunction.

\section{Acknowledgements}

Not applicable.

\section{Funding}

The present study was supported by The Scientific and Technological Project of Shaanxi Province (grant no. 2016SF-246); and The National Nature Science Foundation of China (grant nos. 81670681 and 81870514); and The Major Clinical Research Projects of the First Affiliated Hospital of Xi'an Jiao Tong University (XJTU1AF-CRF-2015-005).

\section{Availability of data and materials}

The datasets used during the present study are available from the corresponding author upon reasonable request.

\section{Authors' contributions}

CD, WX and PT designed the experiments; CD, FH, HX, YW, YL, XD and JZ performed the experiments. MD and XX were responsible for data processing and statistical analyses. YL and $\mathrm{JZ}$ were responsible for writing the manuscript, and XD was responsible for modification of the manuscript. All authors read and approved the manuscript and agree to be accountable for all aspects of the research in ensuring that the accuracy or integrity of any part of the work are appropriately investigated and resolved.

\section{Ethics approval and consent to participate}

All protocols were approved by the Institutional Animal Care and Use Committee of the Xi'an Jiaotong University (Xi'an, China; permit no. XJTULAC2016-558).

\section{Patient consent for publication}

Not applicable.

\section{Competing interests}

The authors declare that they have no competing interests.

\section{References}

1. Nezu M, Souma T, Yu L, Suzuki T, Saigusa D, Ito S, Suzuki N and Yamamoto M: Transcription factor Nrf2 hyperactivation in early-phase renal ischemia-reperfusion injury prevents tubular damage progression. Kidney Int 91: 387-401, 2017.

2. Malek M and Nematbakhsh M: Renal ischemia/reperfusion injury; from pathophysiology to treatment. J Renal Inj Prev 4: 20-27, 2015.

3. Zhu J, Chen X, Wang H and Yan Q: Catalpol protects mice against renal ischemia/reperfusion injury via suppressing PI3K/Akt-eNOS signaling and inflammation. Int J Clin Exp Med 8: 2038-2044, 2015.

4. Kim N, Woo DC, Joo SJ, Song Y, Lee JJ, Woo CW, Kim ST, Hong S, Cho YM and Han DJ: Reduction in renal ischemia-reperfusion injury in mice by a phosphoinositide 3-kinase p110gamma-specific inhibitor. Transplantation 99: 2070-2076, 2015.

5. Meng QH, Liu HB and Wang JB: Polydatin ameliorates renal ischemia/reperfusion injury by decreasing apoptosis and oxidative stress through activating sonic hedgehog signaling pathway. Food Chem Toxicol 96: 215-225, 2016.

6. Ozbilgin S, Ozkardesler S, Akan M, Boztas N, Ozbilgin M, Ergur BU, Derici S, Guneli ME and Meseri R: Renal ischemia/reperfusion injury in diabetic rats: The role of local ischemic preconditioning. Biomed Res Int 2016: 8580475, 2016.

7. Akan M, Ozbilgin S, Boztas N, Celik A, Ozkardesler S, Ergur BU, Guneli E, Sisman AR, Akokay P and Meseri R: Effect of magnesium sulfate on renal ischemia-reperfusion injury in streptozotocin-induced diabetic rats. Eur Rev Med Pharmacol Sci 20: 1642-1655, 2016.

8. Wu SH, Chen XQ, Lü J and Wang MJ: BML-111 Attenuates renal ischemia/reperfusion injury via peroxisome proliferator-activated receptor- $\alpha$-regulated heme oxygenase-1. Inflammation 39: 611-624, 2016.

9. Liu D, Jin F, Shu G, Xu X, Qi J, Kang X, Yu H, Lu K, Jiang S, Han F, et al: Enhanced efficiency of mitochondria-targeted peptide SS-31 for acute kidney injury by pH-responsive and AKI-kidney targeted nanopolyplexes. Biomaterials 211: 57-67, 2019.

10. Hu JB, Li SJ, Kang XQ, Qi J, Wu JH, Wang XJ, Xu XL, Ying XY, Jiang SP, You J and Du YZ: CD44-targeted hyaluronic acid-curcumin prodrug protects renal tubular epithelial cell survival from oxidative stress damage. Carbohydr Polym 193: 268-280, 2018.

11. Kimura T, Takabatake Y, Takahashi A, Kaimori JY, Matsui I, Namba T, Kitamura H, Niimura F, Matsusaka T, Soga T, et al: Autophagy protects the proximal tubule from degeneration and acute ischemic injury. J Am Soc Nephrol 22: 902-913, 2011.

12. Hamzawy M, Gouda SAA, Rashed L, Morcos MA, Shoukry H and Sharawy N: 22-oxacalcitriol prevents acute kidney injury via inhibition of apoptosis and enhancement of autophagy. Clin Exp Nephrol 23: 43-55, 2019.

13. Pang L: Inhibition of COX-2/PGE2/EP4 signaling protects against post-hypoxic apoptosis in $\mathrm{H} 9 \mathrm{C} 2$ cardiomyocytes. Faseb J 30: 2016.

14. Xiao CY, Yuhki K, Hara A, Fujino T, Kuriyama S, Yamada T, Takayama K, Takahata $\mathrm{O}$, Karibe $\mathrm{H}$, Taniguchi $\mathrm{T}$, et al: Prostaglandin E2 protects the heart from ischemia-reperfusion injury via its receptor subtype EP4. Circulation 109: 2462-2468, 2004.

15. Liang X, Lin L, Woodling NS, Wang Q, Anacker C, Pan T, Merchant $M$ and Andreasson K: Signaling via the prostaglandin $E_{2}$ receptor EP4 exerts neuronal and vascular protection in a mouse model of cerebral ischemia. J Clin Invest 121: 4362-4371, 2011.

16. Kuida K, Haydar TF, Kuan CY, Gu Y, Taya C, Karasuyama H, Su MS, Rakic P and Flavell RA: Reduced apoptosis and cytochrome c-mediated caspase activation in mice lacking caspase 9. Cell 94: 325-337, 1998.

17. Wiktorowska-Owczarek A and Owczarek J: The effect of hypoxia on PGE2-stimulated cAMP generation in HMEC-1. Cell Mol Biol Lett 20: 213-221, 2015.

18. Li Y, Zhong D, Lei L, Jia Y, Zhou H and Yang B: Propofol prevents renal ischemia-reperfusion injury via inhibiting the oxidative stress pathways. Cell Physiol Biochem 37: 14-26, 2015. 
19. Zhou S, Sun Y, Zhuang Y, Zhao W, Chen Y, Jiang B, Guo C, Zhang Z, Peng H and Chen Y: Effects of kallistatin on oxidative stress and inflammation on renal ischemia-reperfusion injury in mice. Curr Vasc Pharmacol 13: 265-273, 2015.

20. Cursio R, Colosetti P and Gugenheim J: Autophagy and liver ischemia-reperfusion injury. Biomed Res Int 2015: 417590, 2015.

21. Decuypere JP, Ceulemans LJ, Agostinis P, Monbaliu D, Naesens M, Pirenne J and Jochmans I: Autophagy and the kidney: Implications for ischemia-reperfusion injury and therapy. Am J Kidney Dis 66: 699-709, 2015.

22. Li H, Liu X, Zhu Y, Liu Y and Wang Y: Magnolol derivative $002 \mathrm{C}-3$ protects brain against ischemia-reperfusion injury via inhibiting apoptosis and autophagy. Neurosci Lett 588: 178-183, 2015.

23. Qiao PF, Yao L, Zhang XC, Li GD and Wu DQ: Heat shock pretreatment improves stem cell repair following ischemiareperfusion injury via autophagy. World J Gastroenterol 21: 12822-12834, 2015.

24. Jiang M, Liu K, Luo J and Dong Z: Autophagy is a renoprotective mechanism during in vitro hypoxia and in vivo ischemia-reperfusion injury. Am J Pathol 176: 1181-1192, 2010.

25. Tan KY, Li CY, Li YF, Fei J, Yang B, Fu YJ and Li F: Real-time monitoring ATP in mitochondrion of living cells: A specific fluorescent probe for ATP by dual recognition sites. Anal Chem 89: 1749-1756, 2017.

26. Lewis SC, Uchiyama LF and Nunnari J: ER-mitochondria contacts couple mtDNA synthesis with mitochondrial division in human cells. Science 353: aaf5549, 2016.

27. Lauritzen KH, Kleppa L, Aronsen JM, Eide L, Carlsen H, Haugen ØP, Sjaastad I, Klungland A, Rasmussen LJ, Attramadal $\mathrm{H}$, et al: Impaired dynamics and function of mitochondria caused by mtDNA toxicity leads to heart failure. Am J Physiol Heart Circ Physiol 309: H434-H449, 2015.
28. Cheng N, Lo YS, Ansari MI, Ho KC, Jeng ST, Lin NS and Dai H: Correlation between mtDNA complexity and mtDNA replication mode in developing cotyledon mitochondria during mung bean seed germination. New Phytol 213: 751-763, 2017.

29. Hämäläinen RH: Mitochondria and mtDNA integrity in stem cell function and differentiation. Curr Opin Genet Dev 38: 83-89, 2016.

30. Baruffini E, Ferrari J, Dallabona C, Donnini C and Lodi T: Polymorphisms in DNA polymerase $\gamma$ affect the mtDNA stability and the NRTI-induced mitochondrial toxicity in Saccharomyces cerevisiae. Mitochondrion 20: 52-63, 2015.

31. Sokolowska M, Chen LY, Liu Y, Martinez-Anton A, Qi HY, Logun C, Alsaaty S, Park YH, Kastner DL, Chae JJ and Shelhamer JH: Prostaglandin E2 inhibits NLRP3 inflammasome activation through EP4 receptor and intracellular cAMP in human macrophages. J Immunol 194: 5472-5487, 2015.

32. Chang HH, Young SH, Sinnett-Smith J, Chou CE, Moro A, Hertzer KM, Hines OJ, Rozengurt E and Eibl G: Prostaglandin E2 activates the mTORC1 pathway through an EP4/cAMP/PKAand $\mathrm{EP} 1 / \mathrm{Ca} 2+-$ mediated mechanism in the human pancreatic carcinoma cell line PANC-1. Am J Physiol Cell Physiol 309: C639-C649, 2015.

33. Chang HH, Young S, Sinnett-Smith J, Chou CEN, Hines OJ, Rozengurt E and Eibl G: Abstract 1028: Prostaglandin E2 activates the mTORC1 pathway through an EP4/cAMP/PKA and EP1/calcium-mediated mechanisms in human pancreatic carcinoma cells. Am J Physiol Cell Physiol 309, 2015.

34. Xu S, Ge JP, Zhang ZY and Zhou WQ: AB063. A prostaglandin E (PGE) receptor EP4 is involved in the cell growth and invasion of prostate cancer via the cAMP-PKA/PI3K-AKT signaling pathway. Transl Androl Urol 6 (Suppl 3), 2017. 